\title{
Biodiversidade das Plantas Medicinais: benefícios e riscos
}

\author{
Biodiversity of Medicinal Plants: benefits and risks
}

\author{
Pedro Henrique Silva de Rossi" ${ }^{\bullet}$, Juliana Audi Giannoni ${ }^{\bullet}$, Marie Oshiwa ${ }^{\circ}$, Sandra Maria \\ Barbalho $^{\circ}$, Anna Claudia Sahade Brunatti ${ }^{\circ}$, Luiz Fernando Santos Escouto ${ }^{\circ}$, Andressa Aparecida \\ Jacinto Sampaio $^{\circ}$, Marcelo Martins Pereira ${ }^{\circ}$, Tatiane Barboza da Costa ${ }^{\circ}$
}

Faculdade de Tecnologia de Marília, Marília, São Paulo, Brasil. *Autor para correspondência. E-mail: pps.2010.ph@gmail.com

\begin{abstract}
Resumo: Sabe-se que a biodiversidade contribui significativamente para a subsistência e o desenvolvimento humanos e, portanto, desempenha um papel predominante no bem-estar da população global. Tendo-se assim, as substâncias naturais há muito serviam como fontes de drogas terapêuticas, onde drogas incluindo digitálicos, ergotamina, quinina e salicilatos podem ser citadas como alguns exemplos clássicos, onde as fontes naturais envolvem uma abordagem multifacetada que combina técnicas botânicas, fitoquímicas, biológicas e moleculares. Esta revisão sistemática apresenta como objetivo ilustrar uma visão geral e avaliação de evidências interpostas para com o consumo e conhecimento das plantas medicinais como um todo. A metodologia empregada foi uma pesquisa mista (qualitativa e quantitativa), aplicando uma revisão de literatura e a distribuição de um questionário online para obtenção de resultados fidedignos e de largo espectro para avaliar o conhecimento sobre o assunto. Conclui-se que os dados precocemente impostos, bem como resultados avaliados e interpretados reflete de tal maneira que os entrevistados demonstraram que conhecem e já fizeram uso de plantas medicinais, além de recomendarem o uso e destacar a sua importância.
\end{abstract}

Palavras-chave: biodiversidade, fitoterápicos, plantas medicinais.

\begin{abstract}
It is known that biodiversity contributes to human subsistence and development and, therefore, plays a predominant role in the well-being of the global population. Thus, as natural substances they have long served as sources of therapeutic drugs, where drugs including digitalis, ergotamine, quinine and salicylates can be cited as some classic examples, where natural sources involve a multifaceted approach that combines botanical, phytochemicals, biological and molecular. This systematic review aims to illustrate an overview and evaluation of evidence interposed to the consumption and knowledge of medicinal plants as a whole. The methodology used for a mixed research (qualitative and quantitative), applying a literature review and the distribution of an online questionnaire to obtain reliable and wideranging results to assess the knowledge on the subject. It is concluded that the data imposed early, as well as results and interpreted reflect in such a way that the interviewees demonstrated that they know and have already used medicinal plants, in addition to recommending the use and highlighting its importance.
\end{abstract}

Keywords: biodiversity, phytotherapics, medicinal plants.

\section{Introdução}

Por milênios, a humanidade dependeu muito das plantas para se alimentar e também para o tratamento de doenças. Os produtos naturais sempre contribuíram amplamente para o desenvolvimento da medicina moderna e ainda continuam a desempenhar um papel significativo na descoberta de medicamentos. Ao dar uma olhada na história da modernidade sobre os remédios, encontramos a aplicação de glicosídeos digitálicos, durante o décimo oitavo século, para o tratamento de doenças cardiovasculares, e a casca de salgueiro era popularmente usada para o tratamento da dor e de condições semelhantes à febre (Sen \& Samanta, 2014).

A vasta diversidade genética disponível em plantas, animais e microrganismos apresenta uma riqueza de possibilidades para a melhoria da humanidade na produção de alimentos, materiais e medicamentos (Sen \& Samanta, 2014).

As plantas oferecem um recurso único e renovável para a descoberta de novas biomoléculas terapeuticamente ativas devido às características estruturais e biológicas diversidade de seus constituintes. No entanto, apenas uma pequena fração do reino vegetal ainda foi analisado para seus possíveis usos medicinais. Novas técnicas de química combinatória e triagem de alto rendimento de produtos vegetais também já que o design de novo é agora um pilar para a descoberta de novos medicamentos (Sen \& Samanta, 2014). 
A biodiversidade fornece a suporte para humanos no quesito saúde e bem-estar, fornecendo o básico para os requisitos de vida. A diversidade biótica na genética e componentes bioquímicos foram aproveitados para alimentos que sustentam a vida e fontes medicinais. Nos últimos anos, assistimos ao advento de tecnologias avançadas abordagens para análise molecular e genética detalhes em todo o espectro da vida, incluindo plantas. Isso resultou na catalogação de vastas quantidades de plantas específicas potencialmente perspicazes conhecimento em publicações ou bancos de dados com curadoria (Sarkar, 2009).

Por outro lado, percebe-se uma razão para o consumo de Plantas Medicinais que pode demandar um resultado pouco satisfatório, uma vez que, a suposição de "natural" pode ser igualdo ao "seguro" é certamente um fator de relevância para o consumo. Contudo, está noção é perigosamente enganosa, em virtude dos compostos farmacológicos, que possuem ingredientes ativos, dos quais, alguns podem ser associados como efeitos adversos (Peters, 2009).

O objetivo deste trabalho é ilustrar uma visão geral, revisão sistemática e avaliação de evidências interpostas para com o consumo e conhecimento das plantas medicinais como um todo, uma vez que, os produtos vegetais oferecem um vasto repertório de diversidade química que, por sua vez, pode fornecer uma série de estruturas principais, além dos desafios terapêuticos, a diversidade da química e a biodiversidade (Sen \& Samanta, 2014).

\section{Revisão}

O método misto é definido como o processo de coleta, análise e combinação de técnicas quantitativas e qualitativas onde o principal pressuposto que comprova a racionalidade da abordagem em multimétodo é que a interação entre eles proporciona melhores possibilidades analíticas.

Foi elaborado um questionário, com perguntas simplificadas e de resposta curtas baseadas nos artigos estudados, fazendo uma correlação entre os resultados que pretende-se obter e os resultados finais.

Por meio da plataforma gratuita do Google Forms, foi distribuído de forma online, gratuita e aleatória para vários entrevistados, frisando como registro a idade e o gênero. Composto por onze questões, que variam entre múltipla escolha e dissertativa, a intenção base estava definida de acordo com os materiais encontrados na literatura.

O questionário ficou disponível por um período de oito dias (16 a 23 de fevereiro de 2021) e divulgado pelos meios de comunicação mais comuns e de fácil acesso. Ao final, foram entrevistados 163 indivíduos. Após a entrevista, os dados foram tabulados e analisados de acordo com a proporção de respostas no software Microsoft Excel 2010, em seguida os resultados foram descritos e comparados com os resultados descritos na literatura.

Foram utilizadas as seguintes bases de dados eletrônica: PubMed, Scielo e Lilacs. Os termos de pesquisa foram construídos usando "plantas medicinais" e "fitoterapia".

\section{Discussão}

Ao longo da história, os humanos usaram as plantas para diversos fins, incluindo a fitoterapia. A finalidade e a importância dessas plantas baseiam-se na disseminação do conhecimento desse recurso, que estreita a relação entre o homem e a natureza, disseminando a sabedoria do grande público. Para muitas comunidades, as plantas medicinais são consideradas uma forma alternativa de tratar doenças e até mesmo manter a saúde (Pinto et al., 2006).

Ainda segundo Pinto et al. (2006), embora essa prática seja importante, ainda é ameaçada por diversos fatores, como a conveniência de acesso à medicina moderna e a mudança de pessoas do ambiente natural para as áreas urbanas, o que leva a isso perda do conhecimento popular herdado e disseminado por gerações.

Nesse sentido, essa avaliação de valor científico não está apenas relacionada a comunidade científica que precisa cada vez mais de confirmação do valor terapêutico, os materiais medicinais também são culturalmente ricos para uma nação, Moreira et al. (2002) afirma que a disseminação e realização desse conhecimento sobre as pesquisas de usos terapêuticos das plantas mostram que elas são a ameaça de extinção de inúmeras espécies, muitas das quais ainda são cientificamente desconhecidas.

Foram registrados em maioria, o público feminino $(71,2 \%)$ seguido pelo público masculino $(28,2)$, além do público que não quiseram se identificar de acordo com o gênero $(0,6 \%)$.

De acordo com os anos de nascimento de cada entrevistados obtemos uma média escalonadas entre as décadas de 1950, 1970, 1980 e 1990 o que mais se pronunciaram, interpretando assim que, essa faixa etária fizeram e ainda fazem o consumo de plantas medicinais, e apresentam vasto conhecimento. 
Quando foram perguntados se conheciam sobre o uso de plantas medicinais, obtivemos o resultado esperado, onde $98,8 \%$ apresentavam conhecimento e $83,4 \%$ já fizeram uso de plantas medicinais, dos quais confirmaram utilizar as plantas medicinais para a prevenção e cura de diversas enfermidades (Alves et al., 2015).

Devido à grande variedade de plantas medicinais que nos rodeia, com as mais diversas formulações e propriedades terapêuticas, as plantas mais citadas durante as entrevistas foram: Boldo, Babosa, Camomila, Hortelã, Bálsamo, Erva Cidreira, Carqueja, Arnica, Alecrim, Losna, Bartimão, Alho, Gengibre, Salsinha, Açafrão, Guaco e Erva de Santa Maria (Mastruz).

Entre muitas outras variedades ainda é necessário realizar pesquisas sobre segurança e atributos, além do uso correto e possíveis interações medicamentosas que podem estar presentes nas plantas mencionadas como alternativas de estratégias viáveis para a comunidade ao tratamento doença (Lima \& Fernandes, 2020).

Ao serem perguntados se necessário fosse fazer o uso de um tratamento fitoterápico recomendado por um médico, atendendo os padrões estabelecidos pelos órgão de fiscalização, 90,2\% dos entrevistados fariam o uso das plantas medicinais e também consideram importante, apesar da biodiversidade (quarta maior do mundo) e discrição para mais de mil espécies do ponto de vista demográfico, o Brasil não ter uma infraestrutura para produção e/ou extração racional de plantas medicinais.

Apesar da abundante biodiversidade brasileira e do grande interesse da população pelo o uso da medicina tradicional, atualmente o mercado brasileiro de fitoterápicos ainda é muito modesto, representando cerca de 261 milhões de dólares americanos. Isso representa menos de $5 \%$ do mercado global de medicamentos brasileiros, estimado em cerca de 28 bilhões de dólares americanos em 2014 (Calixto, 2000).

Uma análise cuidadosa das publicações farmacológicas recentes sobre plantas revela que, em geral, os estudos foram realizados com extratos brutos, e apenas alguns artigos investigaram a segurança (toxicologia) e os mecanismos subjacentes de ação. Em contraste, os estudos realizados com ingredientes ativos derivados de plantas trazem algumas noções sobre o mecanismo de ação e são comumente publicadas no revistas científicas de melhor qualidade, e consequentemente, poucos medicamentos fitoterápicos foram desenvolvidos e aprovado pela agência reguladora brasileira ANVISA (Dutra, 2016).

\section{Considerações finais}

Este trabalho proporcionou às pessoas um conhecimento mais profundo sobre a origem da cultura e a prática do uso da fitoterapia. Observou-se que a utilização das propriedades fito condutoras das plantas medicinais no ambiente domiciliar que é de grande importância na vida das pessoas que utilizam a fitoterapia como alternativa para o tratamento de doenças.

O Brasil possui a maior quantidade de biodiversidade do mundo, representando aproximadamente $20-22 \%$ de todas as espécies de plantas conhecidas. Certamente, a área de plantas é uma das os campos de investigação mais relevantes no Brasil, como ecoado pelo grande número de artigos científicos publicados em periódicos científicos revisados por pares.

\section{References}

Alves, J. J. P., Lima, C. C. L., Santos, D. B., \& Bezerra, P. D. F. 2015. Conhecimento popular sobre plantas medicinais e o cuidado da saúde primária: um estudo de caso da comunidade rural de Mendes, São José de Mipibu/RN. Carpe Diem: Revista Cultural e Científica do UNIFACEX, 13(1), 136-156.

Calixto, J. B. 2000. Efficacy, safety, quality control, marketing and regulatory guidelines for herbal medicines (phytotherapeutic agents). Brazilian Journal of Medical and Biological Research, 33(2), 179-189.

Dutra, R. C., Campos, M. M., Santos, A. R., \& Calixto, J. B. 2016. Medicinal plants in Brazil: Pharmacological studies, drug discovery, challenges and perspectives. Pharmacological Research, 112, 4-29.

Lima, B. B., \& Fernandes, F. 2020. Uso e diversidade de plantas medicinais no município de Aracati - CE, Brasil. Journal of Applied Pharmaceutical Sciences, 7, 24-42.

Moreira, R. C. T., Costa, L. C. B., Costa, R. C. S., \& Rocha, E. A. 2002. Abordagem Etnobotânica acerca do Uso de Plantas Medicinais na Vila Cachoeira, Ilhéus, Bahia, Brasil. Acta Farmacéutica Bonaerense, 21(3), 205-211.

Peters, D. 2009. CAM: doing more good than harm. Focus on Alternative and Complementary Therapies, 14(3), 176-178.

Pinto, E. P. P., Amorozo, M. C. M., \& Furlan, A. 2006. Conhecimento popular sobre plantas medicinais em comunidades rurais de mata atlântica- Itacaré, BA, Brasil. Acta Botanica Brasilica, 20(4), 751-762. 
Sarkar, I.N. 2009. Biodiversity Informatics: the emergence of a field. BMC Bioinformatics, 10, S1.

Sen, T., \& Samanta, S. K. 2014. Medicinal Plants, Human Health and Biodiversity: A Broad Review. In: Mukherjee J. (eds) Biotechnological Applications of Biodiversity. Advances in Biochemical Engineering/Biotechnology, vol. 147. Heidelberg: Springer.

\section{Minicurrículo}

Pedro Henrique Silva de Rossi. Discente e Pesquisador do curso de Tecnologia em Alimentos da Faculdade de Tecnologia de Marília (FATEC). Foi nomeado para ocupar cargo público na Câmara Municipal de Marília e Prefeitura Municipal de Marília durante os anos de 2012 à 2016. Atualmente trabalha como Correspondente do Banco Bradesco S/A, em regime CLT. Possui publicação em Revista Nacional e Internacional. É revisor de periódicos internacionais. Possui certificado da Academia Publons sobre a graduação de revisores por pares.

Juliana Audi Giannoni. Pós-Doutorado em Ciência dos Alimentos com Irradiação Gama e Processamento Mínimo pela Universidade Federal de Lavras (UFLA), Lavras/MG, onde lecionou de 2006 a 2008. Doutorado (2004) e Mestrado (2000) em Agronomia/Horticultura pela Universidade Estadual Paulista "Júlio de Mesquita Filho" Campus Botucatu-SP. Graduação em Agronomia (1998) pela Universidade Federal de Lavras (UFLA), Lavras/MG. Professora Associado I, junto ao curso de Tecnologia em Alimentos e da Pós-Graduação da FATEC/Marília/SP. Tem experiência na área de Ciência e Tecnologia de Alimentos, atuando principalmente nos seguintes temas: Toxicologia, Processamento mínimo, Irradiação Gama, Análise sensorial e Tecnologia de Açúcar e Álcool.

Marie Oshiiwa. Possui graduação em Ciências com Habilitação Matemática pela Universidade de Marília (1987), Habilitação em Química pela Faculdade Auxilium de Filosofia Ciências e Letras de Lins (1995), mestrado em Agronomia (Energia na Agricultura) pela Universidade Estadual Paulista Júlio de Mesquita Filho (2001) e doutorado em Agronomia (Agricultura) pela Universidade Estadual Paulista Júlio de Mesquita Filho (2005). Atualmente é docente responsável pelas disciplinas de Estatística, Metodologia da Pesquisa Científica e Trabalho de Conclusão de Curso do curso de Tecnologia em Alimentos; membro do Corpo Editorial da Revista Alimentus e está na Coordenação do curso de pós-graduação Gestão em Controle de Qualidade dos Alimentos na FATEC de Marília.

Sandra Maria Barbalho. Possui Bacharelado em Ciências Biológicas pela Universidade Federal de São Carlos (1992); Licenciatura em Ciências Biológicas pela Universidade Federal de São Carlos (1991), Mestrado em Genética e Evolução pela Universidade Federal de São Carlos (1994) e Doutorado em Ciências pela Universidade Federal de São Carlos (1999). Atualmente é docente do Curso de Medicina da Universidade de Marília, UNIMAR. É professora de Bioquímica da Faculdade de Tecnologia em Alimentos de Marília (FATEC). Possui experiência em pesquisa e orientação na área Interdisciplinar envolvendo Bioquímica, Metabolismo, Plantas Medicinais e Alimentos Funcionais na Reabilitação da Saúde. Atua também como pesquisadora na área de metabolismo em doenças metabólicas (Síndrome Metabólica e Doenças Cardiovasculares). Possui 152 artigos publicados em periódicos (nacionais e internacionais). Participou de mais 80 bancas julgadoras. É autora de seis capítulos de livro (um nacional e 5 internacionais). Revisora de 28 periódicos internacionais. Total de citações no GOOGLE SCHOLAR: 1094, ÍNDICE H: 17 e ÍNDICE i10: 31.

Anna Claudia Sahade Brunatti. Graduada em Nutrição pela Universidade de Marília (1998), Mestre em Agronomia pela Universidade de Marília (2008) e Doutora em Agronomia pela UNESP de Botucatu (2016). No ano de 2006 iniciou suas atividades como Professor de Ensino Superior pelo Centro Paula Souza na FATEC, curso Superior em Tecnologia de Alimentos. Apresenta experiência na área de Ciência e Tecnologia de Alimentos, com ênfase em Tecnologia de Produtos de Origem Vegetal. 
Luiz Fernando Santos Escouto. Possui graduação em Nutrição pela Faculdade de Nutrição do Instituto Metodista de Educação e Cultura (1986), mestrado em Agronomia (Energia na Agricultura) pela Universidade Estadual Paulista Júlio de Mesquita Filho (2000) e doutorado em Agronomia (Energia na Agricultura) pela Universidade Estadual Paulista Júlio de Mesquita Filho (2004). A partir de 2007 assume a docência/pesquisa na FATEC no curso de Tecnologia em Alimentos é titular da disciplina Análise Sensorial.

Andressa Aparecida Jacinto Sampaio. Graduando em Tecnologia em Alimentos pela Faculdade de Tecnologia de Marília.

Marcelo Martins Pereira. Graduando em Tecnologia em Alimentos pela Faculdade de Tecnologia de Marília.

Tatiane Barboza da Costa. Graduando em Tecnologia em Alimentos pela Faculdade de Tecnologia de Marília.

Como citar: Rossi, P.H.S., Giannoni, J.A., Oshiwa, M., Barbalho, S.M., Brunatti, A.C.S., Escouto, L.F.S., Sampaio, A.A.J., Pereira, M.M., \& Costa, T.B. 2021. Biodiversidade das Plantas Medicinais: benefícios e riscos. Pubsaúde, 5, a139. DOI: https://dx.doi.org/10.31533/pubsaude5.a139

Recebido: 2 mar. 2021.

Revisado e aceito: 25 mar. 2021.

Conflito de interesse: os autores declaram, em relação aos produtos e companhias descritos nesse artigo, não ter interesses associativos, comerciais, de propriedade ou financeiros que representem conflito de interesse.

Licenciamento: Este artigo é publicado na modalidade Acesso Aberto sob a licença Creative Commons Atribuição 4.0 (CC-BY 4.0). 